OPEN ACCESS

Edited by:

Hailin Tang,

Sun Yat-sen University Cancer Center

(SYSUCC), China

Reviewed by:

Wei Xiong,

Central South University, China

Chang Li,

Soochow University, China

*Correspondence:

Yong Zhou

wzgxy3yy@163.com

${ }^{\dagger}$ These authors have contributed equally to this work

Specialty section:

This article was submitted to Molecular and Cellular Oncology,

a section of the journal

Frontiers in Oncology

Received: 25 March 2020 Accepted: 14 September 2020

Published: 18 November 2020

Citation:

Wang Z, Deng M, Chen L, Wang W, Liu G, Liu D, Han Z and Zhou Y (2020) Circular RNA Circ-03955 Promotes Epithelial-Mesenchymal Transition in Osteosarcoma by Regulating miR3662/Metadherin Pathway.

Front. Oncol. 10:545460. doi: 10.3389/fonc. 2020.545460

\section{Circular RNA Circ-03955 Promotes Epithelial-Mesenchymal Transition in Osteosarcoma by Regulating miR-3662/Metadherin Pathway}

\author{
Zhengguang Wang ${ }^{1 \dagger}$, Mingsi Deng ${ }^{2 \dagger}$, Liangjian Chen ${ }^{2}$, Weiguo Wang ${ }^{1}$, Gengyan Liu ${ }^{1}$, \\ Dongbiao Liu ${ }^{1}$, Zhipeng Han ${ }^{1}$ and Yong Zhou ${ }^{1 *}$ \\ ${ }^{1}$ Department of Orthopaedics, The Third Xiangya Hospital, Central South University, Changsha, China, ${ }^{2}$ Department of \\ Stomatology, The Third Xiangya Hospital, Central South University, Changsha, China
}

Osteosarcoma is the most common primary malignant tumor, especially in children and adolescents. Circular RNAs (circRNAs) are found to play roles in the progression of osteosarcoma. However, the exact functions of circRNAs in osteosarcoma development still need to be clarified. We obtained differentially expressed circRNAs and miRNAs from a GSE99671 data set (GEO database). The gene co-expression network of ceRNAs and osteosarcoma-related genes was analyzed using the STRING database. qRT-PCR was used to detect the expression of circ-03955 and miR-3662. Transwell assays and flow cytometry were performed to detect phenotypic changes in cell function. A xenograft tumor model was established using BALB/c nude mice. Dual luciferase activity and RNA immunoprecipitation assays were performed to assess the relationship between circ-03955, miR-3662, and metadherin (MTDH). Immunohistochemistry, immunofluorescence, and Western blotting were used to assess protein expression levels. Circ-03955 was significantly upregulated, and miR-3662 was downregulated in osteosarcoma. Circ-03955 silencing inhibited the growth and metastasis of osteosarcoma. Mechanism analysis revealed that circ-03955 could bind to miR-3662, and the latter could target MTDH, leading to its suppressed expression and facilitating epithelial-mesenchymal transition (EMT). All these findings demonstrate that the presence of circ-03955 promotes EMT in osteosarcoma by acting as miR-3662 sponge-mediated MTDH expression.

Keywords: circ-03955, miR-3662, osteosarcoma, metadherin, epithelial-mesenchymal transition

\section{INTRODUCTION}

Osteosarcoma is the most common malignant bone neoplasm, especially in children and adolescents. It has a high rate of mortality and disability $(1,2)$ as well as high levels of metastasis and invasion. At present, the treatment of osteosarcoma usually involves a combination of chemotherapy and surgical resection. The five-year survival rate of non-metastatic osteosarcoma patients is $60 \%-70 \%$ (3), but the five-year survival rate of patients with distant metastasis is only $20 \%$ (4). Therefore, it is important to explore the molecular mechanisms of osteosarcoma 
occurrence and metastasis, find molecular biomarkers for early diagnosis, and identify an effective molecular basis for the development of novel therapeutic targets in order to inhibit the occurrence of osteosarcoma metastasis.

Circular RNA (circRNA) is a relatively newly identified form of RNA. The 3' and 5' ends of circRNA are linked together, forming a closed ring structure, and circRNA is abundant in the eukaryotic transcriptome. In 2012, Salzman (5) found that many highly expressed circRNAs are associated with their corresponding linear RNA, which can form multiple homotypes of circular RNA by "non-classical splicing," and speculated that the expression of circRNA might be a general pattern of gene expression program in human cells. There is increasing evidence that abnormal circRNA plays an important role in tumorigenesis $(6,7)$, tumor development $(8,9)$, and metastasis $(10,11)$. Chen confirms that circ-100782 is highly expressed in pancreatic ductal adenocarcinoma and regulates BxPC3 cell proliferation through the IL6-STAT3 pathway (12). Studies show that circRNA can regulate the proliferation and apoptosis of osteosarcoma and are good targets for clinical diagnosis (13-15). However, the effect of circRNA on osteosarcoma has not been studied in detail.

Current studies show that miRNAs can be involved in the regulation of gene expression and can complement the transcripts of target genes, thus playing an important role in the occurrence and development of tumors (16, 17). The biological function of circRNA is related to that of miRNAs. A regulatory network is formed by competitive inhibition. Li shows that circ- 0001785 regulates the pathogenesis of osteosarcoma by sponging miR-1200, which upregulates HOXB2 expression (18). Song indicates that hsa_circ-0001564 acts as an miR-29c-3p sponge to mediate the tumorigenicity of osteosarcoma (19). More details about the function of ceRNA in osteosarcoma still need to be elucidated.

In this study, we first screened circRNA (circ-03955) from the GEO database (GSE99671 data set) and find that circ-03955 was highly expressed in osteosarcoma. We also find that circ-03955 knockdown could inhibit the migration, invasion, and epithelialmesenchymal transition (EMT) of osteosarcoma. In addition, circ-03955 acts as a ceRNA for miR-3662 to regulate the progression of metadherin (MTDH)-mediated osteosarcoma.

\section{MATERIALS AND METHODS}

\section{Patient Tissue Specimens}

Forty samples of osteosarcoma tissue and 40 cases of adjacent non-osteosarcoma tissues were obtained from the Department of the third Xiangya Hospital of Central South University. All tissue specimens were removed during scheduled surgery. After rinsing the tissue specimens with saline, the specimens were cut into small pieces and frozen in liquid nitrogen. The specimens were then stored at $-80^{\circ} \mathrm{C}$ for subsequent RNA extraction experiments. The use of normal bone tissue specimens and osteosarcoma bone tissue specimens in all patients was evaluated and approved by the Ethical Committee of the third Xiangya Hospital of Central South University. All participants provided written informed consent after receiving information about the design of the study.

\section{Cell Culture and Transfection}

A human osteoblastic cell line, OB3, and the human osteosarcoma cell lines Saos-2, MG-63, and U2OS were purchased from the American Type Culture Collection (ATCC, Rockville, USA). Saos-2 and U2OS cells were cultured in McCoy's 5a medium modified (ATCC, USA), but 15\% fetal bovine serum was added to Saos-2 growth medium, and 10\% fetal bovine serum was added to MG-63 growth medium. MG-63 cells were cultured in Eagle's minimum essential medium (ATCC, USA) containing 10\% heat-inactivated fetal bovine serum. The circ-03955 interference vectors sh-circ-03955-1 and sh-circ-03955-2 and the circ-03955 overexpression vector pCDNA3.1-circ-03955 (OE-circr-03955) were obtained from GenePharma (Shanghai, China). A non-targeting shRNA (shNC) or the empty pcDNA3.1vector (OE-NC) were used as negative controls. The miR-3662 mimics and miR-3662 inhibitor were purchased from GeneCopoeia (Guangzhou, China). The MTDH mRNA interference vector sh-MTDH was obtained from GenePharma (Shanghai, China). All transfections were performed using Lipofectamine 6000 reagent (Life Technologies, USA) according to the manufacturer's protocols.

\section{Bioinformatic Analysis}

The GSE99671 data set, which included 18 pairs of osteosarcoma tissue specimens and paired normal bone tissue specimens, from the GEO database (https://www.ncbi.nlm.nih.gov/geo) was analyzed for differential gene expression. Gene expression was calculated using $|\log 2 \mathrm{FC}|>1$ and FDR $<0.05$ as the criteria for significant difference using the $\mathrm{R}$ statistics package limma. The miRanda package was used to predict the miRNAs that can bind MTDH and circRNA, and then the possible ceRNA network of differentially upregulated circRNA, differentially downregulated miRNAs, and MTDH was constructed. The first 20 genes reported to be associated with osteosarcoma were selected from the DisGeNET database for co-expression analysis with the ceRNA network maps. Cytoscape 3.6.1 was used to build the network graph.

\section{Quantitative Reverse Transcription- Polymerase Chain Reaction Assay}

The linear validation of circRNA was performed in the osteosarcoma cell line MG-63. MG-63 cells were extracted using Trizol reagent (Invitrogen, California, USA), and circRNA was linearized by RNase R. Then, the expression of circular RNA was detected using qRT-PCR assay. The specific steps were carried out as previously described (20). SYBR Green I fluorescent dye was used in the qRT-PCR assays. The relative expression of these genes was calculated with triplicate experiments using the $2^{-\Delta \Delta C T}$ equation. The following primers were used for $\mathrm{qRT}$-PCR detection. Primer mRNA (Forward: 5'TGCTCCACTGACTGTTGT-3'; Reverse: 5' -TTGGC ATTTCTCCTCTAA-3'), primer circ-03955 (Forward: 5'ATTAGAGGAGAAATGCCAAGG-3'; Reverse: 5'-AGCC CATCTGCAACAACAG-3'), primer miR-3662 (Forward: 5'- 
CGCTCACAGTTACACTTCTT-3'; Reverse: 5'-GTGCTT CATCAGTCACTACTCATC-3'), U6 (Forward: 5'-CTCGCTT CGGCAGCACA-3'; Reverse: 5'-AACGCTTCAGGAA TTTGCGT-3'), and the internal reference primer GAPDH (Forward: 5'-CCTTCCGTGTTCCTAC-3'; Reverse: 5' GACAACCTGGTCCTCA-3'). All primers were designed and synthesized by Sangon Biotech (Shanghai, China).

\section{Cell Proliferation}

Cells were digested with $0.25 \%$ trypsin and suspended in basic culture medium containing $10 \%$ fetal bovine serum to form a single-cell suspension. The single-cell suspension was inoculated into 96 -well plates at a concentration of $1 \times 10^{5} /$ well. The culture plate was placed in an incubator at $37^{\circ} \mathrm{C}$ containing $5 \% \mathrm{CO}_{2}$ and saturated humidity. The suspension of cells was centrifuged at $2000 \mathrm{rpm}$ for 15 minutes. The supernatant was dissolved and crystallized by adding $150 \mu \mathrm{L}$ DMSO. The cells were transfected for 24 hours by enzyme-linked immunosorbent assay (Bio-Rad, USA). The OD value at $570 \mathrm{~nm}$ was detected after 24,48 , and 72 hours of transfection.

\section{Cell Apoptosis}

The cells were digested with $0.25 \%$ trypsin, and then the digested cells were combined into a single-cell suspension $\left(1 \times 10^{6} / \mathrm{mL}\right)$ in basic culture medium containing $10 \%$ fetal bovine serum. After centrifugation with $1000 \mathrm{rpm}$ for five minutes, the culture medium was discarded, the cells washed with precooled PBS (Solarbio, Beijing, China) and centrifuged at $1000 \mathrm{rpm}$ for 5 minutes. The cells were resuspended in $100 \mu \mathrm{L}$ of binding buffer $\left(1 \times 10^{5} / \mathrm{mL}\right)$, lightly blended with $5 \mu \mathrm{L}$ Annexin V-FITC at room temperature for 10 minutes and then incubated with $5 \mu \mathrm{L}$ $\mathrm{PI}$ at room temperature for 5 minutes. PBS was then added to $500 \mu \mathrm{L}$, and the apoptotic rate was detected immediately after blending.

\section{Cell Migration and Invasion}

Serum was removed to starve the cells for 24 hours, and $0.25 \%$ trypsinase was added to digest the cells. After termination of digestion, the cells were washed twice with precooled PBS and then suspended in serum-free medium containing BAS, and the cell concentration was adjusted to $5 \times 10^{5} / \mathrm{mL}$. A $100-\mu \mathrm{L}$ cell suspension was added into Transwell chambers, and $600 \mu \mathrm{L}$ of medium containing $10 \%$ FBS was added into a 24 -well plate subchamber. After 24 hours of culture in a constant temperature incubator with $5 \% \mathrm{CO}_{2}$ and saturated humidity of $37^{\circ} \mathrm{C}$, the Transwell chamber was removed, and the culture medium in the well was washed twice with PBS and then fixed with methanol for 30 minutes. The upper chamber liquid was removed, and the cells dyed with $0.1 \%$ crystal violet dye solution for 20 minutes. The untransferable cells were wiped on the bottom membrane surface of the upper chamber with a wet cotton rod and washed three times with PBS. Finally, the chamber membrane was observed at $100 \mathrm{X}$ magnification. The difference between the invasion and migration assays was that 1 $\mathrm{mg} / \mathrm{mL}$ of Matrigel (BD, NJ, USA) was added to the bottom of the upper chamber and incubated in a $37^{\circ} \mathrm{C}$ incubator for 4 hours after dilution by $100 \mu \mathrm{L}$. The gelatinized material was then removed and reserved.

\section{Xenograft Assay}

Ten 4- to 5-week old SPF male BALB/c nude mice were purchased from Auragene (Changsha, China). Nude mice were randomly divided into an sh-NC group and an sh-circ-03955 group with five mice in each group. Osteosarcoma MG-63 cells transfected with sh-circ-03955 and sh-NC plasmids were administered by subaxillary injection to form tumors at a cell concentration of $2 \times 10^{5}$ cells. After the osteosarcoma MG-63 cells were injected into the mice, the tumor volume was measured using calipers every five days. Six weeks later, the mice were euthanized, and the tumors were weighed. The study was approved by the medical ethics committee of the third Xiangya Hospital, central South University.

\section{Immunofluorescence}

Logarithmic-stage growth cells of different treatment groups were washed twice with PBS and then used to prepare cell smears. The cells were fixed at room temperature with $4 \%$ paraformaldehyde for 30 minutes, washed twice with PBS, and then treated at room temperature with $0.1 \%$ Triton-X 100 for 3 minutes to make them permeable. After washing the permeate, $5 \%$ skimmed milk powder was added and incubated at room temperature for 2 hours. Anti-E-cadherin antibody (1:300, ab76055, Abcam) and anti-N-cadherin antibody (1:200, ab98952, Abcam) was added and the reaction incubated overnight at $4^{\circ} \mathrm{C}$. After the first antibody was washed out, fluorescently labeled goat antimouse IgG antibody (1:200, ab150115, Abcam) was added and incubated at room temperature for 1 hour, then washed with PBST three times. After adding DAPI staining and washing out the staining solution the cells were observed under the fluorescence microscope (Olympus, Japan).

\section{Western Blotting}

Tissues or cells were removed, and then total protein was extracted using RIPA buffer. BCA protein concentration kits (Sigma-Aldrich, USA) were used to determine the protein concentration. SDS sample buffer was added, and the reaction mixture was placed in a $95^{\circ} \mathrm{C}$ water bath for 5 minutes, then centrifuged after cooling. Electrophoresis using 10\% SDS-PAGE gels was performed with $20 \mu \mathrm{g}$ protein per well. After the completion of electrophoresis, the protein obtained was transferred to PVDF transfer membranes using a wet rotation method. The protein was sealed at room temperature for one hour with 5\% BSA (Solarbio, Beijing, China). Then antibodies to MTDH (1:1000, 9596, CST), E-cadherin (1:1000, 3195T, Cell Signaling Technology), N-cadherin (1:1000, 13116T, Cell Signaling Technology), Vimentin (1:1000, 5741T,Cell Signaling Technology), GAPDH (1:500, ab8245, Abcam), and $\beta$-actin (1:500, ab8226, Abcam) were added to the membrane. After incubation overnight at $4^{\circ} \mathrm{C}$, the membranes were washed three times with TBST buffer. Membranes were incubated at room temperature for 30 minutes with goat antimouse IgG antibody 
labeled with horseradish peroxidase and then washed with TBST buffer three times. Color was developed using immunoblotting chemiluminescence reagent ECL (Auragene, Changsha, China) and then detected using a gel imaging system.

\section{Immunohistochemistry}

Immunohistochemistry (IHC) was performed to evaluate changes in the expression levels of E-cadherin and N-cadherin. The xenograft tissue sections of the sh-NC and sh-circ-03955 groups were taken out and completely dewaxed and hydrated. The antigens were repaired by microwave. Then, the sections were washed with PBS three times. The sections were immersed in $3 \% \mathrm{H}_{2} \mathrm{O}_{2}$-methanol solution for 15 minutes at room temperature to block endogenous peroxidase and then washed with PBS three times. E-cadherin (1:500, GB12082, Servicebio) and N-cadherin (1:800, GB11135, Servicebio) were added and incubated in a $37^{\circ} \mathrm{C}$ water bath for one hour, followed by postblocking incubation at room temperature for 30 minutes and then washing with PBS three times. The second antibody labeled with horseradish peroxidase was incubated at room temperature for 30 minutes and washed with PBS five times. After adding $\mathrm{DAB}$ for two minutes, the dyeing was terminated, re-dyed with hematoxylin for three minutes, and then sealed with transparent xylene and dripped with neutral gum. Under the light microscope, positive brown staining was observed at a magnification of $200 \times$.

\section{Dual Luciferase Activity Assay}

MG-63 cells were transfected with Wt-circ-03955 plasmid and NC mimics or miR-3662 mimics, according to the instructions accompanying the Lipofectamine 6000 transfection reagent. Mut-circ-03955 plasmid and mimics of NC or miR-3662 were co-transfected, and lysate was added 48 hours later. The supernatant was centrifuged for 10 minutes at $4^{\circ} \mathrm{C}$ at 12,000 $\mathrm{rpm}$, and the luciferase activity was detected using a DualLuciferase ${ }^{\circledR}$ Reporter Assay System (Promega, Wisconsin, USA). MTDH binding to miR-3662 was detected in a similar way.

\section{RNA Immunoprecipitation (RIP) Assay}

MG-63 cells $\left(2 \times 10^{7}\right)$ were collected and added to the same volume of RIP lysate to lyse cells. The supernatant was centrifuged at 12,000 rpm for 10 minutes. In accordance with the instructions of the RIP ${ }^{\mathrm{TM}}$ RNA binding protein immunoprecipitation reaction kit, $900 \mu \mathrm{L}$ of RIP immunoprecipitation buffer containing RNase inhibitor, protease inhibitor, and DNase and $100 \mu \mathrm{L}$ of cell lysate were added into EP tubes containing magnetic beads. IgG antibody or Ago2 antibody were added and incubated overnight at $4^{\circ} \mathrm{C}$ and centrifuged at $12,000 \mathrm{rpm}$. After centrifugation for 10 minutes, the supernatant was discarded and washed six times with $500 \mu \mathrm{L}$ RIP wash buffer. The RNA was purified immediately, and then dissolved and purified in $15 \mu \mathrm{L}$ of DEPC water, then stored at $-80^{\circ} \mathrm{C}$. The positive control (input) group had no antibody, and the experimental group had Ago2 antibody. The expression of circ-03955 and microRNA-3662 in the input group was detected using qRT-PCR assay.

\section{Statistical Analysis}

SPSS19.0 statistical software and GraphPad Prism 6.0 software were used to analyze all the data. Student's $t$-test was used to analyze the differences between the two groups. One-way ANOVA was used to analyze the differences between more than two groups. All of the experimental data are presented as mean \pm standard deviation (SD). Chi-square tests were used to analyze the correlations between the expression of miR3662 and circ-03955 in osteosarcoma cells. The $P$ value was evaluated to determine statistical significance with a $P$ value of less than 0.05 taken to indicate that the difference was statistically significant.

\section{RESULTS}

\section{Screening of miR-3662 and circ-03955 by Bioinformatic Analysis}

To screen differentially expressed miRNA and circRNA in osteosarcoma, we performed bioinformatic analysis on data from the GSE99671 data set, comprising 18 pairs of osteosarcoma tissue specimens and paired normal bone tissue specimens. We identified nine downregulated miRNAs (Table 1) and 13 upregulated circRNAs (Figure 1A) compared with normal bone tissue. Then, we used the miRanda software to predict differentially downregulated miRNAs in osteosarcoma bone samples that can interact with $\mathrm{MTDH}$, including miRNA563 and miR-3662 (Figure 1B). The miRanda software was also used to predict eight miRNAs that could bind to significantly upregulated circRNA and were differentially downregulated in osteosarcoma bone samples. Cytoscape 3.6.1 was used to construct a network map (Figure 1C). The DisGeNET database was used to select the first 20 reported osteosarcoma-related genes: CHEK2, TP53, RB1, VEGFA, EGFR, RUNX2, MDM2, MMP2, MET, DHFR, TNFRSF11A, JUN, RFC1, RECQL4, GSTP1, NR1I2, TOPORS, CYP3A4, MYC, and KCNH1. The ceRNA network map was analyzed using co-expression analysis, and the results show that MTDH and circ-03955 play an important role in the graph (Figure 1D). The expression of circ-03955, MTDH, and miR-3662 was found to be inversely correlated in osteosarcoma tissues (Figure 1E).

TABLE 1 | Differential expression of miRNAs in 18 osteosarcoma bone samples and 18 paired normal bone samples.

\begin{tabular}{lcc}
\hline Gene ID & log2FC & pvalue \\
\hline miR1296 & -1.616 & 0.00053 \\
LET7G & -1.191 & 0.01551 \\
miR4273 & -0.832 & 0.04439 \\
miR568 & -0.880 & 0.02349 \\
miR3662 & -0.925 & 0.04806 \\
miR604 & -0.956 & 0.04127 \\
miR4324 & -1.439 & 0.00352 \\
miR650 & -1.236 & 0.00543 \\
miR223 & -1.087 & 0.02862
\end{tabular}


A

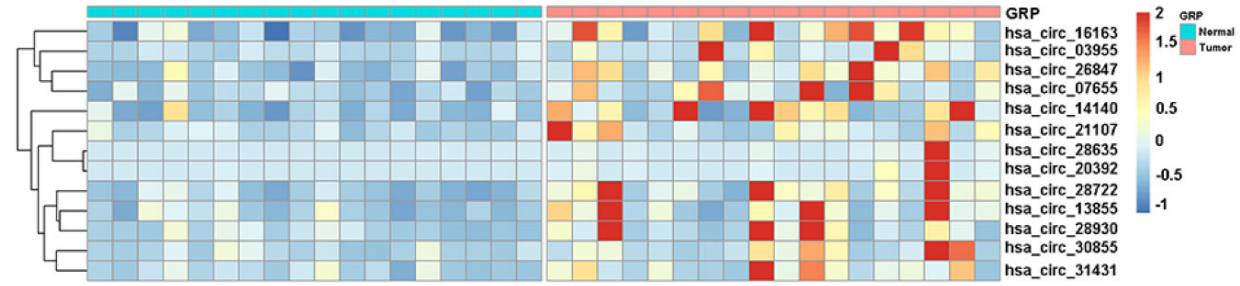

B

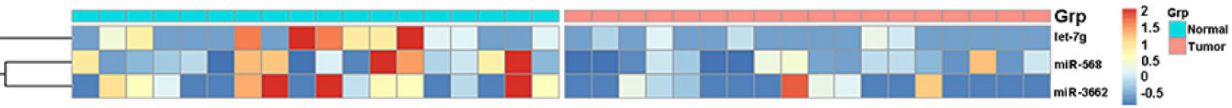

c

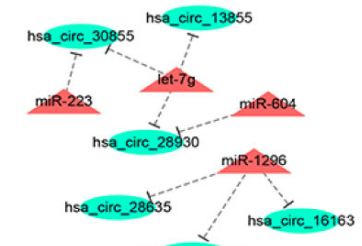

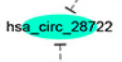<smiles>[C+]1[CH]C1</smiles>

hsa_circ_31931<smiles>C1CC1</smiles>

hse_circ_14740- - miR-650<smiles>C1C2CC1C2</smiles>

E hsa_circ_20392
D
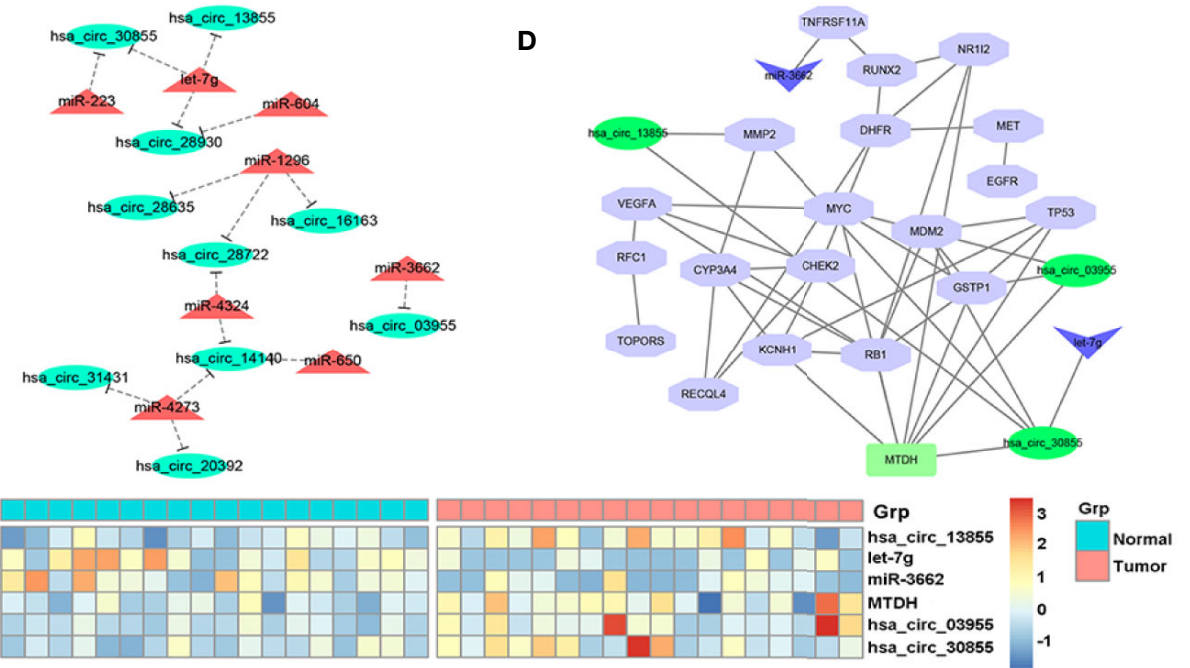

$\mathbf{F}$

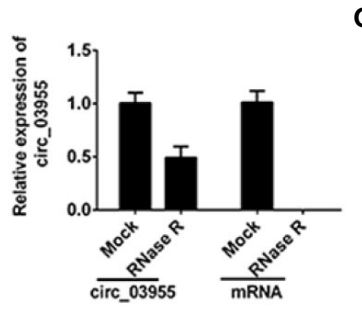

G

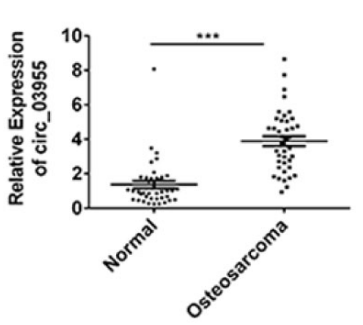

H

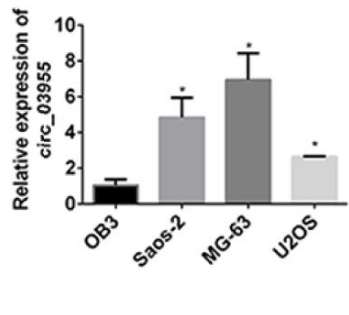

I

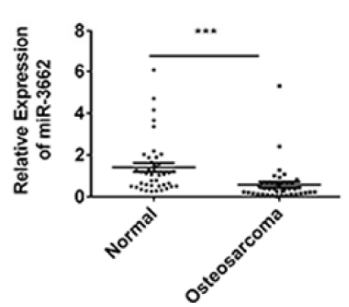

J

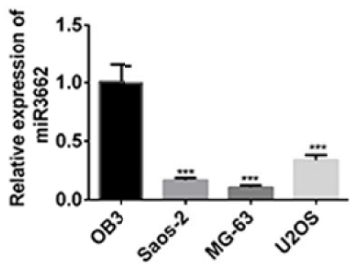

FIGURE 1 | circ-03955 was markedly increased in osteosarcoma cells and tissues. (A) Thermal map of circRNAs that are differentially expressed in the GSE99671 data set. (B) Thermal map of the miRNAs that regulate MTDH expression and are differentially downregulated in the GSE99671 data set. (C) Network map constructed using the GSE99671 data set showing significantly upregulated circRNA-bound miRNAs and downregulated miRNAs. (D) The first 20 genes associated with osteosarcoma were screened by the DisGeNET database for co-expression analysis with ceRNA network maps. (E) Thermograph of gene expression in the ceRNA network of Fig 1D, which was analyzed using GSE99671. (F) The expression of circ-03955 and mRNA detected by qRT-PCR in MG-63 cells treated with RNase R. (G) The expression of circ-03955 detected by qRT-PCR in 40 samples of osteosarcoma tissue specimens and 40 samples of adjacent non-osteosarcoma tissue specimens. (H) Expression of circ-03955 detected by qRT-PCR in normal osteoblasts OB3 in three osteosarcoma cell lines: Saos-2, MG-63, and U2OS. (I) The expression of miR-3662 detected by qRT-PCR in 40 samples of osteosarcoma tissue specimens and 40 samples of adjacent non-osteosarcoma tissue specimens. (J) Expression of miR-3662 detected by qRT-PCR in normal osteoblasts OB3 and three osteosarcoma cell lines: Saos-2, MG-63, and U2OS. Data are presented as the mean \pm standard deviation of three independent experiments, ${ }^{*} p<0.05,{ }^{* \star *} p<0.001$ vs. normal group or OB3 group. 


\section{High Expression of circ-03955 in Osteosarcoma Cells and Tissues}

To verify the differential expression of circ-03955 and miR-3662 in osteosarcoma tissues and cells, we first linearized them using RNase R in MG-63 cells, and then detected the expression of circ-03955. The results show that circ-03955 is resistant to RNase $\mathrm{R}$ digestion, and linear circ-03955 is easy to degrade. They further confirm that circ-03955 is circular (Figure 1F). Previous bioinformatics analysis showed that circ-03955 was highly expressed in osteosarcoma tissue. In this study, we measured the expression of circ-03955 in 40 pairs of osteosarcoma tissues and normal tissues as well as in osteosarcoma cells and normal osteoblasts. The results show that the expression of circ-03955 is significantly increased in osteosarcoma bone tissue compared with adjacent nonosteosarcoma tissue (Figure 1G). The same results were also found in cells in which the expression of circ-03955 in osteosarcoma cells from the Saos-2, MG-63, and U2OS cell lines was significantly higher than that in normal osteoblast cells OB3 (Figure 1H). MG-63 cells had the highest levels. The expression of miRNA-3662 in osteosarcoma tissue was significantly lower than that in adjacent non-osteosarcoma tissue (Figure 1I), and the same result was also found in cells (Figure 1J).

\section{circ-03955 Knockdown Suppressed Osteosarcoma Cells Progression In Vivo and In Vitro}

To verify the effect of circ-03955 on osteosarcoma, we constructed a circ-03955 interference expression vector and transfected it into MG-63 and U2OS cells (Figure 2A). Subsequently, the proliferation of MG-63 and U2OS cells in the sh-circ-03955 group decreased significantly compared with the sh-NC group $(P<0.001)$ (Figure 2B). Compared with the sh-NC group, the apoptotic rate of MG-63 and U2OS cells in the sh-circ-03955 group was significantly higher $(P<0.001)$ (Figure 2C). Compared with the sh-NC group, the migration of MG-63 and U2OS cells in the sh-circ-03955 group was significantly decreased $\left.{ }^{* * *} P<0.001\right)$ (Figure 2D). Compared with the sh-NC group, the invasion of MG-63 and U2OS cells in the sh-circ03955 group was significantly decreased $(P<0.001, P<0.001)$ (Figure 2E).

Assays in xenograft mice showed that circ-03955 knockdown not only suppressed tumor volume, but also inhibited tumor weight, suggesting a suppressor role for circ-03955 knockdown on osteosarcoma tumor growth (Figure 2F). Overall, these results indicate that circ-03955 silencing could suppress the osteosarcoma cells progression in vivo and in vitro.

\section{circ-03955 Knockdown Inhibited the Occurrence of EMT in Osteosarcoma}

Previous studies have shown that circ-03955 knockdown could inhibit the proliferation, apoptosis, invasion, and migration of osteosarcoma. In order to further investigate the effects of circ03955 on EMT on osteosarcoma, we measured the expression of E-cadherin and $\mathrm{N}$-cadherin in circ-03955 knockdown tissues and cells. Compared with sh-NC, E-cadherin was obvious in the cytoplasm of the sh-circ-03955 group. N-cadherin was also expressed at lower levels in the cytoplasm of sh-circ-03955 (Figure 2G). The Western blot results confirmed that, compared with sh-NC, the expression of E-cadherin was markedly increased in the sh-circ-03955 group, and the expression of $\mathrm{N}$-cadherin was significantly decreased (all $P<$ 0.001) (Figure 2H). The same results were also found in tissues. The immunohistochemical results show that the intracellular deposition of E-cadherin protein in the sh-circ-03955 group was significantly higher than that in the sh-NC group, and the intracellular deposition of $\mathrm{N}$-cadherin protein was significantly reduced (Figure 2I). Western blotting also confirmed that, compared with sh-NC, the expression of E-cadherin was increased, and N-cadherin was decreased in sh-circ-03955 (all $P<0.001$ ) (Figure 2J). These results indicate that circ-03955 knockdown could effectively inhibit the EMT of osteosarcoma in vivo and vitro.

\section{miR-3662 Mimics Inhibit Osteosarcoma Cell Proliferation, Promote Cell Apoptosis, and Inhibit Cell Invasion}

Previous studies show that the expression of miRNA-3662 is low in osteosarcoma tissues and cells (Figures 1I, J). To verify the role of miRNA-3662 in the development of osteosarcoma, miRNA-3662 mimics and inhibitor were transfected into osteosarcoma cells MG63 and U2OS (Figure 3A). MTT results show that, compared with NC mimics, the proliferation of MG-63 and U2OS cells in the miR-3662 mimics group was significantly decreased $(P<0.001, P<$ 0.01) (Figure 3B). Flow cytometry showed that, compared with the mimics NC group, the apoptotic rate of MG-63 and U2OS cells in the miR-3662 mimics group was significantly increased $(P<0.001)$ (Figure 3C). Transwell test results showed that, compared with the mimics NC group, the invasion number of the MG-63 and U2OS cells in the miR3662 mimics group decreased significantly $(P<0.001)$ (Figure 3D). These results indicate that miR-3662 mimics could effectively inhibit the proliferation and invasion and promote the apoptosis of osteosarcoma.

\section{circ-03955 Acts as a ceRNA by Sponging miR-3662 and Indirectly Regulates MTDH Expression}

Previous analyses confirm that miR-3662 is the target gene of circ03955, and MTDH is the target gene of miR-3662. In order to verify the results of the bioinformatics analysis, we carried out follow-up studies. qRT-PCR results showed that circ-03955 was expressed primarily in the cytoplasm (Figure 4A). The expression of circ-03955 was negatively correlated with that of miR-3662 ( $r=$ $-0.625, P=0.0042)$. To verify the effect of circ-03955 on the expression of miR-3662 and MTDH, OE-circ-03955 or sh-circ03955 were transfected into MG-63 cells. The qRT-PCR and Western blot results showed that circ-03955 overexpression can inhibit the expression of miR-3662 and promote the expression of MTDH. The knockdown of circ-03955 was shown to promote the 
A

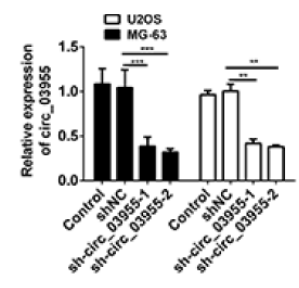

C
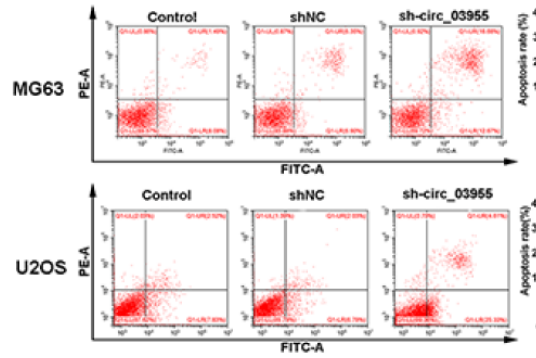

E

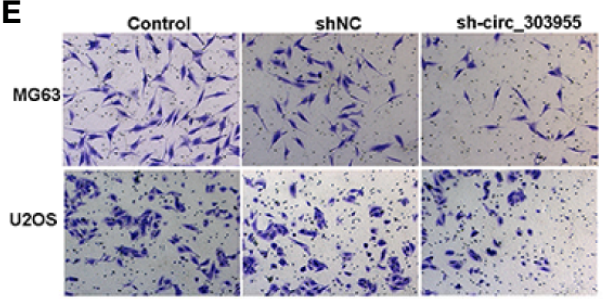

B
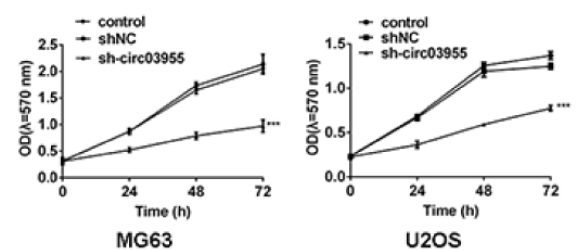

D
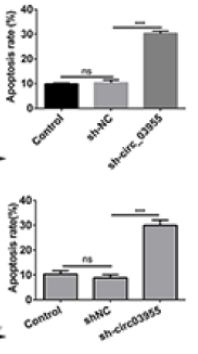

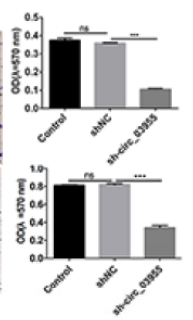

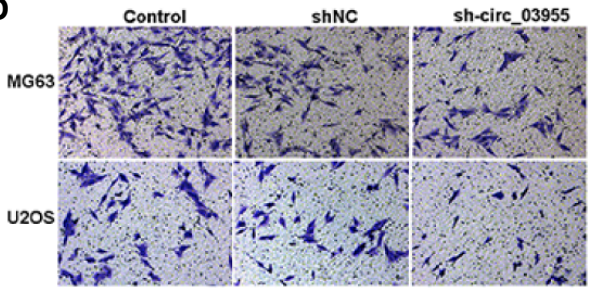

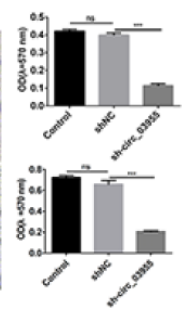

$\mathbf{F}$
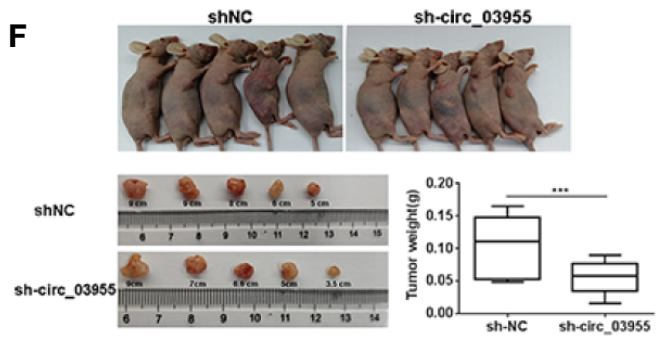

G

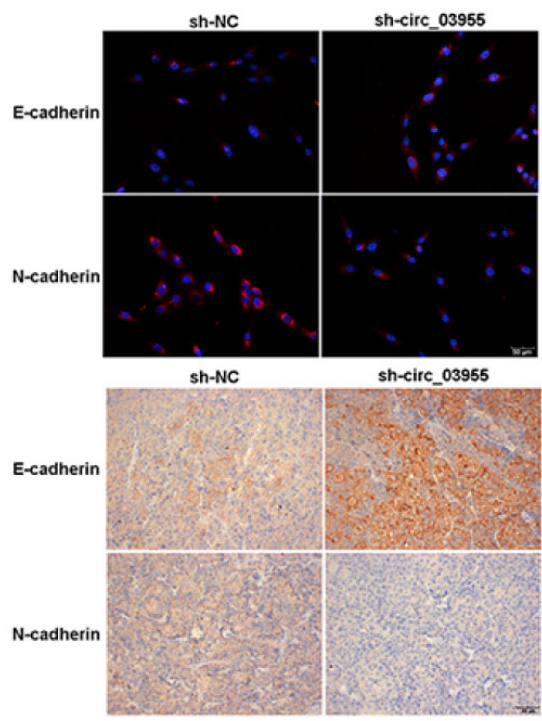

H

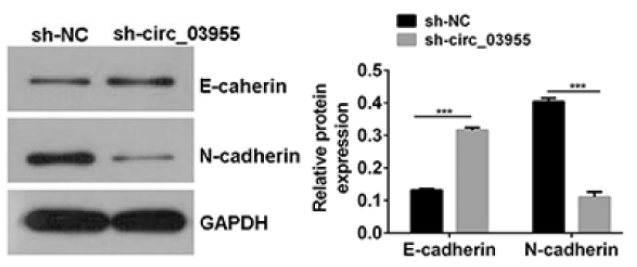

J

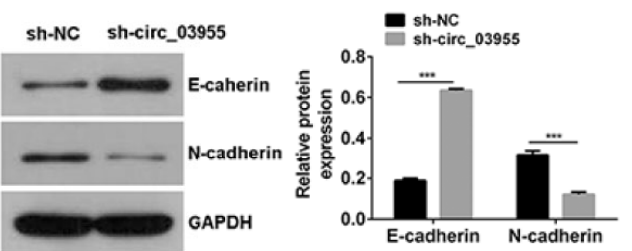

FIGURE 2 | Circ-03955 silencing suppressed osteosarcoma cells progression in vivo and in vitro. (A) qRT-PCR was used to verify whether sh-circ-03955 was successfully transfected into osteosarcoma cells in MG-63 and U2OS. (B) MTT assay was used to detect the effects of sh-circ-03955 on the proliferation of osteosarcoma cells in MG-63 and U2OS at 24, 48, and 72 hours after transfection. (C) Flow cytometry was used to detect the effect of sh-circ-03955 on the apoptosis of osteosarcoma cells in MG-63 and U2OS 48 hours after transfection. (D) Transwell assay was used to detect the effects of sh-circ-03955 on the migration of osteosarcoma cells in MG-63 and U2OS at 48 hours. (E) Transwell assay was used to detect the effect of sh-circ-03955 on invasion of osteosarcoma cells in MG-63 and U2OS at 48 hours. (F) Size and weight of transplanted tumors. (G) The expression of E-cadherin and N-cadherin in osteosarcoma MG-63 cells transfected with sh-NC and sh-circ-03955 for 48 hours was detected by immunofluorescence. (H) Western blotting was used to detect the expression of E-cadherin and N-cadherin in osteosarcoma MG-63 cells transfected with sh-NC and sh-circ-03955 for 48 hours. (I) After transfection of osteosarcoma MG-63 cells with sh-NC and sh-circ-03955, the tumorigenesis experiment was carried out in nude mice, and the transplanted tumor tissues were removed and immunohistochemical assays were performed. (Scale bar $=500 \mu \mathrm{m}, 200 \times$ ) (J) After transfection of osteosarcoma MG-63 cells with sh-NC and sh-circ-03955, the tumorigenesis experiment was carried out in nude mice, and the transplanted tumor tissues were removed for Western blot detection. Data are presented as the mean \pm standard deviation of three independent experiments, NS, not significant, ${ }^{\star \star} p<0.01,{ }^{\star \star \star} p<0.001$ vs. shNC group. 


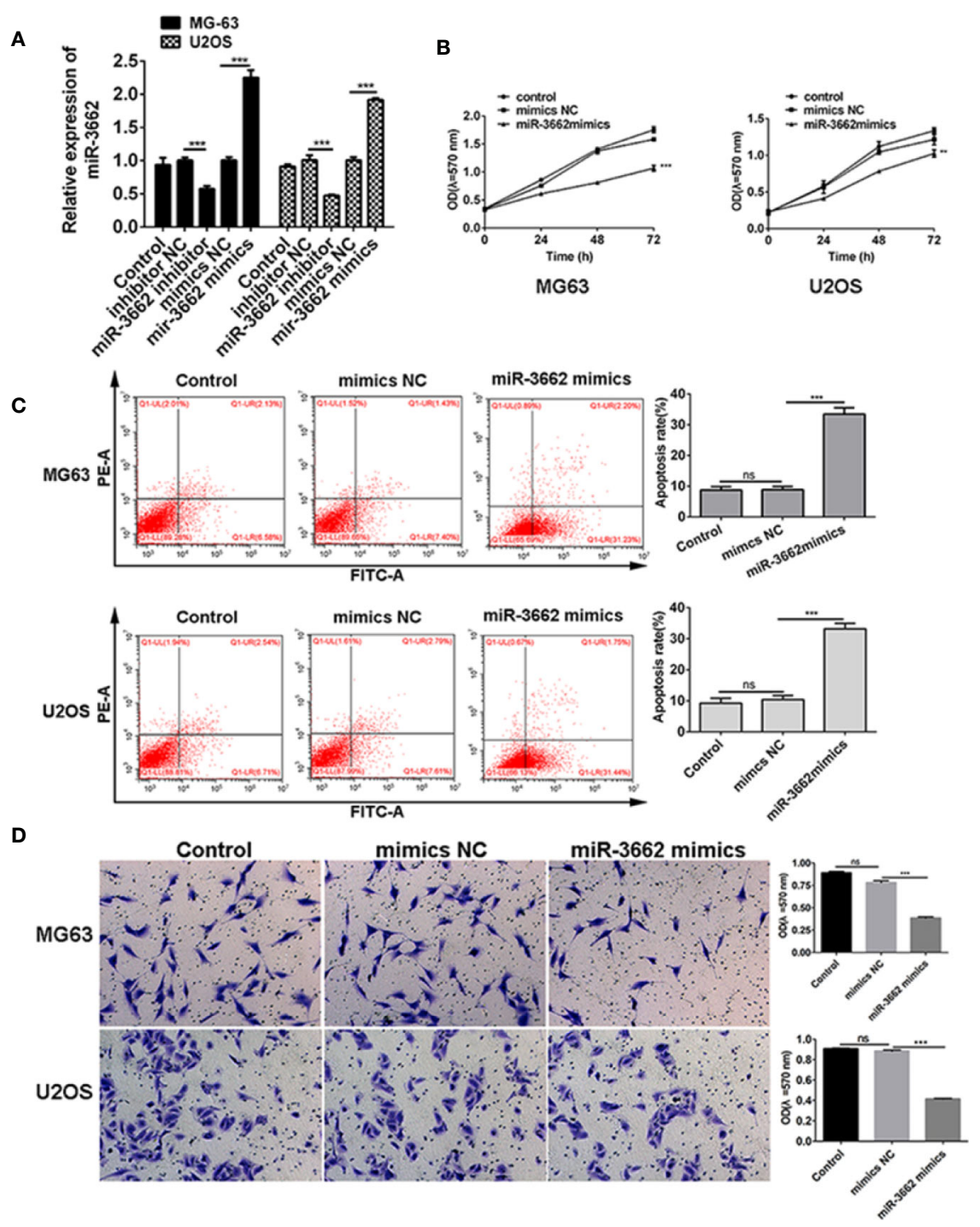

FIGURE 3 | miR-3662 mimics inhibit osteosarcoma cell proliferation, promote cell apoptosis, and inhibit cell invasion. (A) qRT-PCR was used to verify the transformation efficiency of miR-3662 mimics and inhibitor in osteosarcoma cells MG-63 and U2OS. (B) MTT assay was used to detect the effects of miR-3662 mimics on the proliferation of osteosarcoma cells MG-63 and U2OS at 24, 48 and 72 hours after transfection. (C) Flow cytometry was used to detect the effect of miR-3662 mimics on apoptosis of osteosarcoma cells MG-63 and U2OS at 48 hours after transfection. (D) Transwell assay was used to detect the effects of miR3662 mimics on the invasion of osteosarcoma cells MG-63 and U2OS at 48 hours after transfection. Data are presented as the mean \pm standard deviation of three independent experiments, ns, not significant, ${ }^{\star \star} p<0.01,{ }^{* \star *} p<0.001$ vs. shNC group.

expression of miR-3662 and inhibit the expression of MTDH (Figures 4C, D). miR-3662 mimics can also inhibit the expression of MTDH, whereas miR-3662 inhibitor can promote the expression of MTDH in osteosarcoma cells from the MG-63 and U2OS cell lines (Figure 4E). To further investigate the relationships between circ-03955 and miR-3662 and miR-3662 and MTDH a dual luciferase reporting system was used, and the results showed that circ-03955 was directly targeted by miR-3662 (Figure 4F), and MTDH was directly targeted by miR-3662 (Figure 4G) in MG-63 cells.

Ago2, as an effector protein of RISC, participates in miRNA assembly and degradation. Therefore, when the Ago2 protein was pulled down, the circRNA and miRNAs bound to RISC were also pulled down in the RIP assay (PMID: 30999843). The RIP test was performed with Ago2 as the antibody to research the relationship between circ-03955 and miR-3662. We found that circ-03955 and miR-3662 were preferentially enriched in the Ago2 pellet relative to control IgG immunoprecipitates, and endogenous circ-03955 pull-down was specifically enriched in miR-3662-transfected cells (Figure 4H). Knockdown of circ03955 inhibited the increase of MTDH expression induced by the miR-3662 inhibitor (Figure 4I). These results suggest that circ-03955 functions as a ceRNA by sponging miR-3662 and indirectly regulating $\mathrm{MTDH}$ expression. 

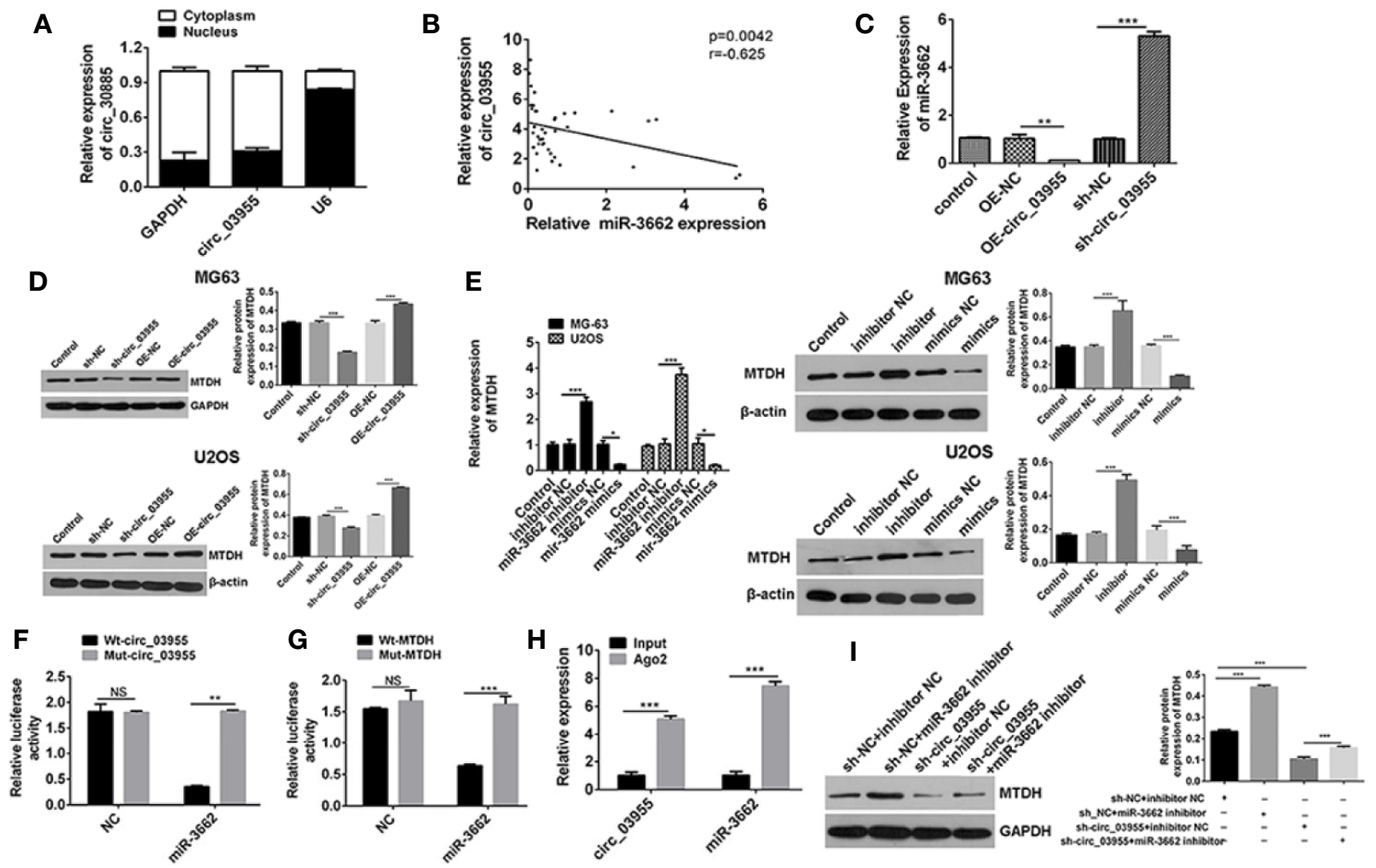

FIGURE 4 | Circ-03955 acts as a ceRNA by sponging miR-3662 and indirectly regulates MTDH expression. (A) The differential expression of circ-03955 in MG-63 cytoplasm and nucleus of osteosarcoma cells verified by qRT-PCR. (B) Analysis of the correlation between circ-03955 and miR-3662 expression in osteosarcoma. (C) qRT-PCR was used to detect the effect of OE-circ-03955 and sh-circ-03955 on the expression of miR-3662 in osteosarcoma MG-63 cells 48 hours after transfection. (D) Western blotting was used to detect the effects of OE-circ-03955 and sh-circ-03955 on the expression of MTDH protein in osteosarcoma cells MG63 and U2OS at 48 hours after transfection. (E) The expression of MTDH in osteosarcoma MG-63 and U2OS was detected by qRT-PCR after 48 hours of transfection. Western blotting was used to detect the expression of MTDH protein in osteosarcoma MG-63 and U2OS at 48 hours after transfection. (F) The binding relationship between circ-03955 and miR-3662 was detected by the dual luciferase reporting system. (G) The binding relationship between miR-3662 and MTDH was detected by the dual luciferase reporting system. (H) Ago2-RIP assays were used to detect the relationship between circ-03955 and miR-3662. (I) Western blot assays were used to verify that circ-03955 reverses the inhibitory effect of miR-3662 on MTDH. Data are presented as the mean \pm standard deviation of three independent experiments, NS, not significant, ${ }^{*} p<0.05,{ }^{* *} p<0.01,{ }^{* \star *} p<0.001$.

\section{miR-3662 Can Reverse the Effects of circ-03955 or MTDH on Osteosarcoma Apoptosis and EMT}

Previous studies show that circ-03955 can inhibit the progression of osteosarcoma and the occurrence of EMT and regulate MTDH expression via miRNA-3662. It is reported that MTDH can promote the occurrence of EMT in osteosarcoma (21). To explore whether circ-03955 exerts its functions by miR-3662/ MTDH in osteosarcoma, rescue experiments were performed. Downregulation of MTDH significantly enhanced cell apoptosis and inhibited cell migration and invasion, and the effect could be abolished by the miR-3662 inhibitor (Figures 5A-C). Downregulation of $\mathrm{MTDH}$ also significantly inhibited Vimentin and $\mathrm{N}$-cadherin expression and promoted the expression of E-cadherin, an effect that could be inhibited by the miR-3662 inhibitor (Figure 5D). Downregulation of circ03955 significantly promoted the apoptosis of MG63 cells and inhibited cell migration and invasion, also effects that could be inhibited by the miR-3662 inhibitor (Figures 6A-C).
Downregulation of circ-03955 markedly inhibited the expression of Vimentin and $\mathrm{N}$-cadherin and promoted the expression of E-cadherin, and the effect could be inhibited by miR-3662 inhibitor (Figures 6D, E). These results indicate that circ-03955 contributes to EMT by suppressing miR-3662.

\section{DISCUSSION}

Osteosarcoma ranks third in the incidence of malignant tumors in children and adolescents and in mortality rate ranks first (22, 23 ). In the past few decades, despite improvements in surgical and adjuvant radiotherapy techniques, the five-year survival rate of osteosarcoma patients with metastasis or recurrence has not improved, remaining at about $20 \%(24,25)$. Due to the low five-year survival rate of osteosarcoma, it is important to explore new treatment programs for clinical application. Recently, circRNA has been shown to play an important role in the progression of many diseases and appears to be 
A

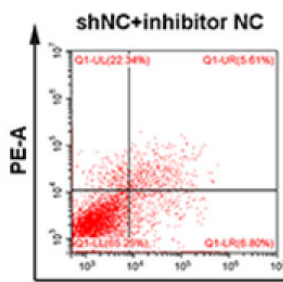

shNC+
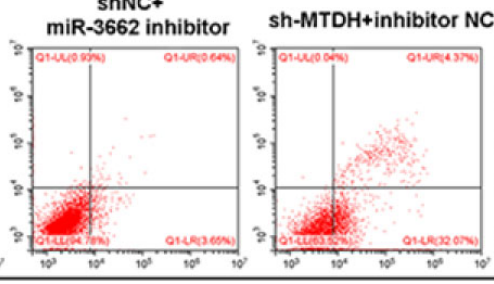

FITC-A

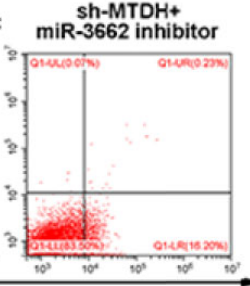

sh-MTDH

B
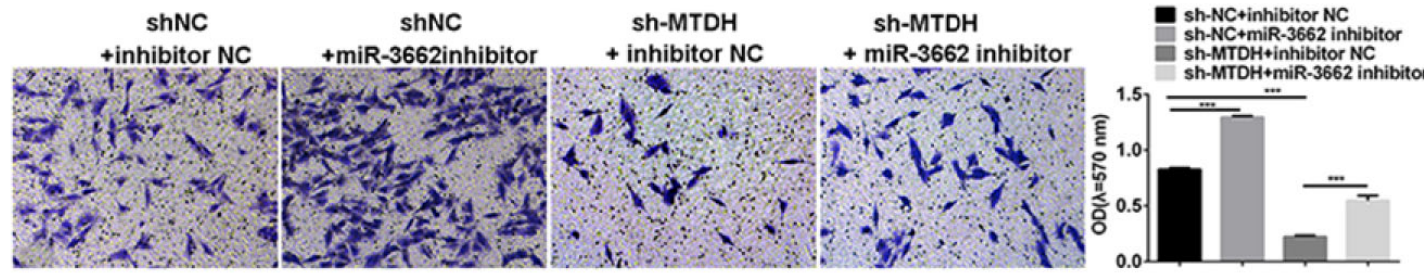

c

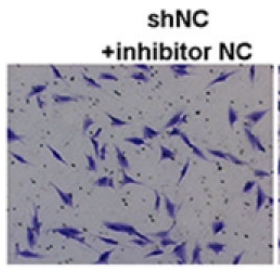

shNC

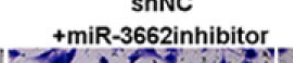

sh-MTDH + inhibitor NC 78 :
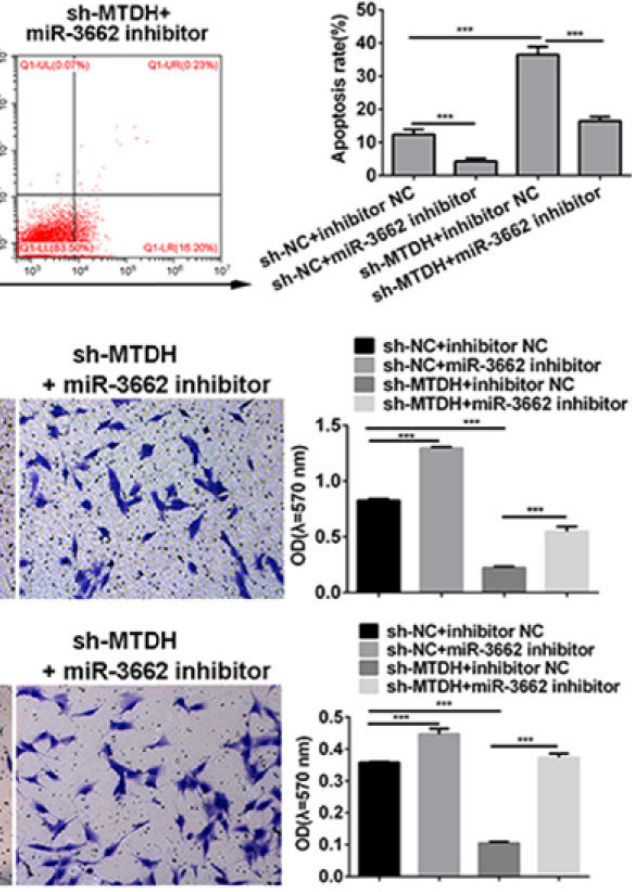

tinhibitor NC h-MTDH+inhibitor NC
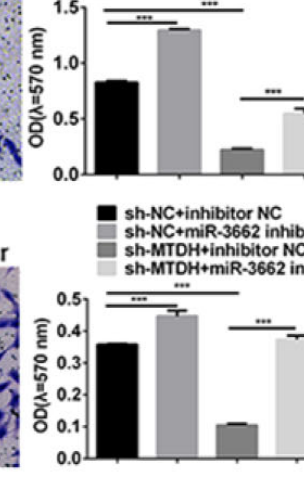
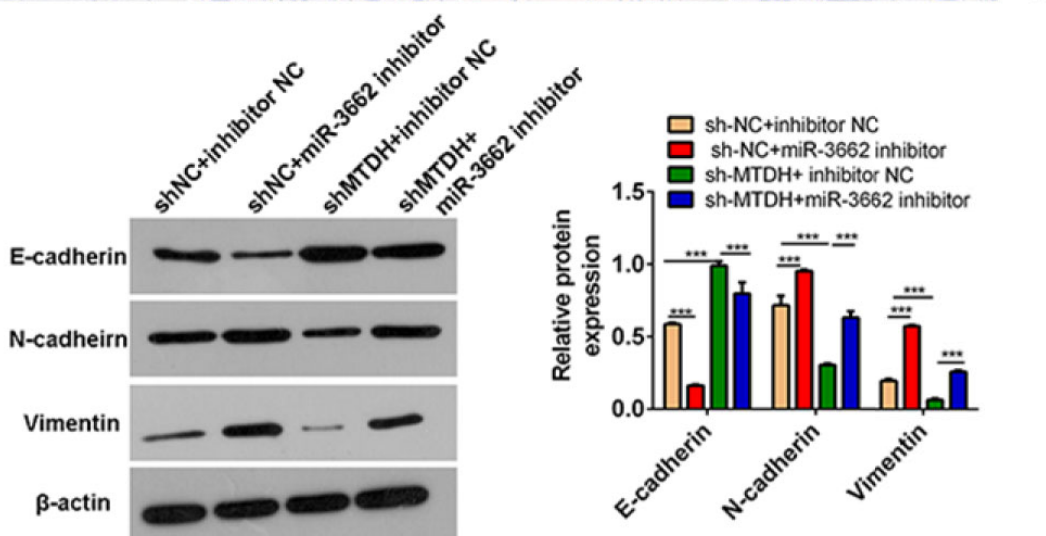

FIGURE 5 | miR-3662 inhibitor rescues the inhibitory effect of shMTDH on osteosarcoma cells. (A) Flow cytometry was used to detect the apoptotic rate of the four groups in the recovery experiment and to verify that miR-3662 could reverse the apoptotic effect of MTDH on osteosarcoma cells. (B) Transwell assays were used to detect the migration of four groups in the recovery experiment and to verify that miR-3662 could reverse the migration effect of MTDH on osteosarcoma cells.

(C) Transwell assay was used to detect the invasion of the four groups in the recovery experiment and to verify that miR-3662 could reverse the invasion effect of MTDH on osteosarcoma cells. (D) Western blotting was used to detect the expression of EMT-related proteins (E-cadherin, N-cadherin, and Vimentin) in four groups and to verify that miR-3662 could reverse the effect of MTDH on the EMT of osteosarcoma cells. Data are presented as the mean \pm standard deviation of three independent experiments, ${ }^{\star \star \star} p<0.001$.

abnormally expressed in various types of tumor, providing a potential novel therapeutic target for tumor treatment (26-28). In this study, we identified a novel circRNA (circ-03955) and confirmed that circ-03955 was upregulated in osteosarcoma tissues and cells. Circ-03955 knockdown inhibited the proliferation, migration, and invasion of osteosarcoma cells from the MG63 and U2OScell lines and promoted apoptosis. Knockdown of circ-03955 inhibited the progression of osteosarcoma in a mouse model. These results suggest that circ03955 acts as a tumor promotor in osteosarcoma.
With the increase in circRNA research, some circRNA-related bioinformatics databases have emerged. The CircNet database reports that 212,950 circRNAs have been found. In the nc2Cancer database, 172 circRNA have been reported to be related to 31 malignant tumors. Based on these databases, some novel circRNAs have been screened out by bioinformatic methods, and their function in tumors has been verified. Liu (29) used the CircNet database to establish that the expression of circ-ZEBl.5, circ-ZEBl.19, circ-ZEB1.17, and circ-ZEB 1.33 in lung cancer was lower than that in normal tissues. It has been demonstrated that 
A

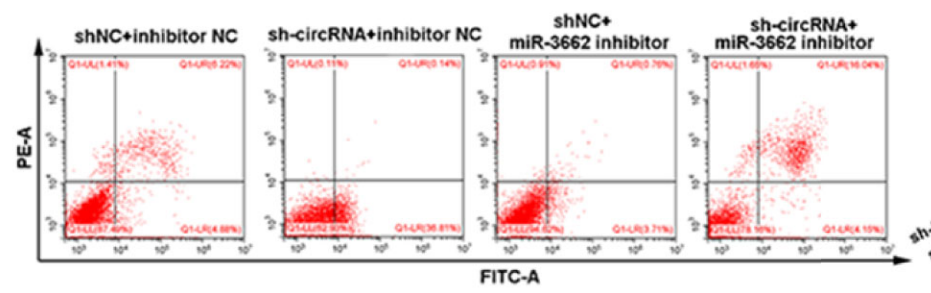

$R-3662$ inhibitor

A

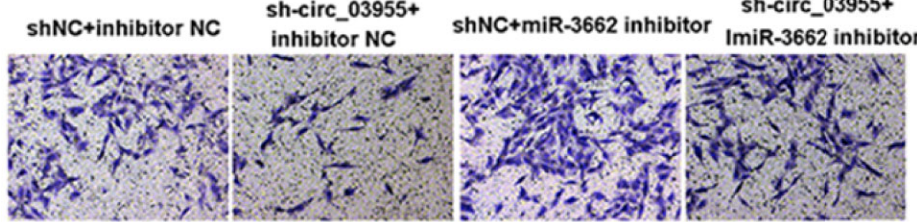

C
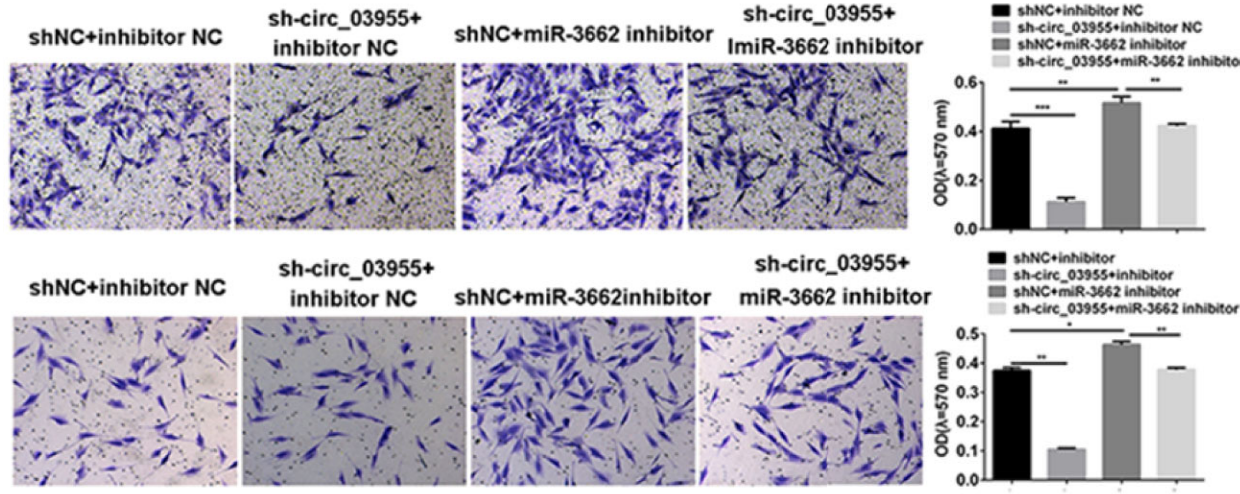

D

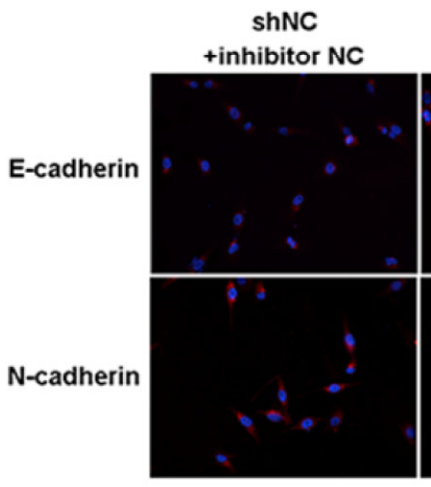

sh-circRNA

sh-NC

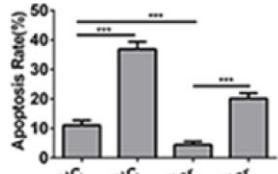

$$
0
$$

$$
\text { or }{ }^{2} \mathrm{C}
$$
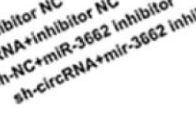

+ miR-3662 inhibito

\section{sh-circRNA}
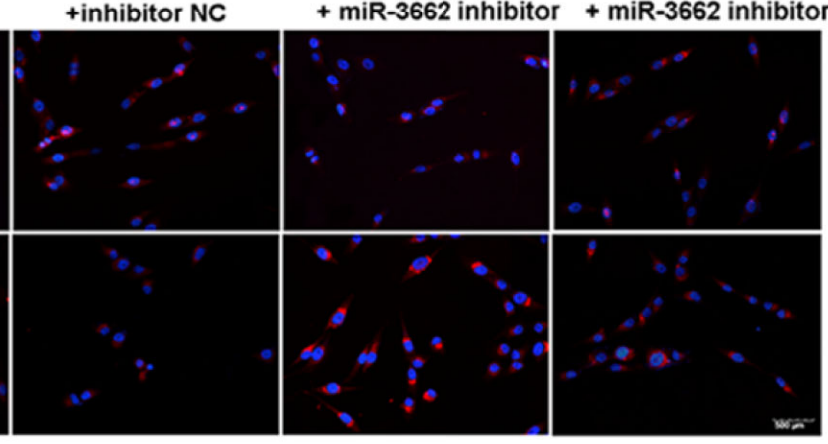

E
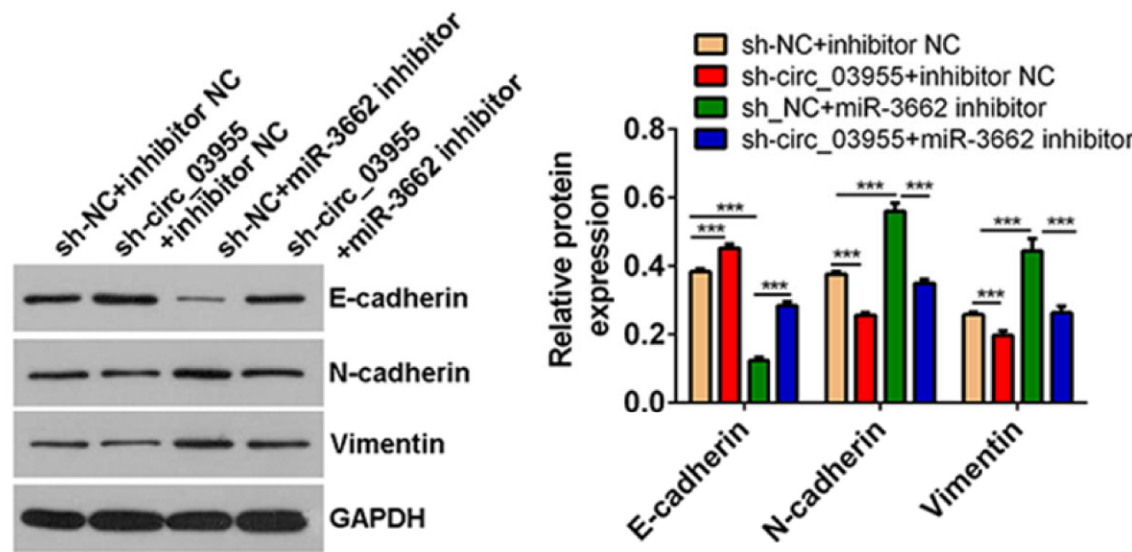

FIGURE 6 | shcirc_03955 rescues the inhibitory effect of miR-3662 on osteosarcoma cells. (A) Flow cytometry was used to detect the apoptotic rate of the four groups in the recovery experiment and to verify that miR-3662 could reverse the apoptotic effect of circ-03955 on osteosarcoma cells. (B) Transwell assay was used to detect the migration of four groups in the recovery experiment and to verify that miR-3662 could reverse the migration effect of circ-03955 on osteosarcoma cells. (C) Transwell assay was used to detect the invasion of the four groups in the recovery experiment and to verify that miR-3662 could reverse the invasion effect of circ-03955 on osteosarcoma cells. (D) The expression of E-cadherin and N-cadherin proteins in four groups was detected by immunofluorescence assay. (Scale bar $=500 \mu \mathrm{m})$. (E) Western blotting was used to detect the expression of EMT-related proteins (E-cadherin, N-cadherin, and Vimentin) in four groups and to verify that miR-3662 could reverse the effect of circ-03955 on the EMT of osteosarcoma cells. ${ }^{\star \star \star} P<0.001$ indicated that the difference has statistical significance. Data are presented as the mean \pm standard deviation of three independent experiments, ${ }^{\star} p<0.05,{ }^{* *} p<0.01,{ }^{\star \star *} p<0.001$. The red color indicates the expression of the target protein, and the blue color indicates the nuclear staining. The magnification is $200 x$. 
circ-EIF3J, circ-PAIP2, and circ-FUNDCl are significantly overexpressed in cervical cancer cells (30). In this study, we identified a differentially highly expressed circ-03955 form GSE99671 data set using bioinformatics methods. Circ-03955 is a novel circular RNA, located in chr8 of hg19 with a genomic length of 286 bp. However, no studies have suggested that circ-03955 contributes to any disease, including osteosarcoma. We found that circ-03955 was circular RNA using qRT-PCR after RNase R treatment. We propose for the first time that circ-03955 is a promotor of osteosarcoma. We also found that circ-03955 has a marked effect on the EMT of osteosarcoma. Thus, circ-03955 was established as a circRNA with the potential to be a target for further experiments. miR-3662, which is a target of circ-03955, was shown to be significantly decreased in osteosarcoma. A previous study showed that miR-3662 is downregulated in acute myeloid leukemia, whereas it is increased in lung adenocarcinoma (31). However, the role of miR-3662 and related mechanisms were unclear in osteosarcoma. Our study showed that miR-3662 was downregulated in osteosarcoma, and cell function was contrary to circ-03955. We explored the interactions between circ-03955, miR3662, and MTDH, which is the target of miR-3662.

In 2013, Hansen (32) and Memczak (33) found that circRNA can act as a "sponge" to block and competitively inhibit miRNAs in tumors, so circRNA can participate in the pathogenesis of tumors as an endogenous competitive RNA. There are more than 60 conservative binding sites on the sequence of circular RNA-7 (ciR-7) binding to miRNA-7, and they are abundant in the cytoplasm. They can bind to more than 20,000 miRNAs-7 in cells, thus affecting the transcriptional inhibition function of miRNA-7 in cells $(33,34)$. CircRNA SRY has been found to have six binding sites, thus acting as a sponge for miRNA-138 (35). Studies show that circRNA can competently inhibit miRNAs to participate in the signaling pathway of tumors $(36,37)$. There are many studies that have proposed a sponge mechanism between circRNA and miRNA. Our results indicate that there is a sponge mechanism between circ-03955 and miR-3662. We found that the expression of circ-03955 and miR-3662 was negatively correlated in clinical samples of osteosarcoma tissues and cells. Circ- 03955 could bind to miR-3662, and miR-3662 could bind to MTDH. circ-03955 and miR-3662 regulate the effect of MTDH on osteosarcoma through endogenous competition. Our study confirmed that circ-03955 acts as a sponge to competitively inhibit miR-3662 in osteosarcoma, and downstream target MTDH was regulated by miR-3662. This is consistent with the idea that circRNA can participate in the signaling pathway of tumors through competitive inhibition of miRNAs.

Recent studies have shown that MTDH could promote lung metastasis of breast cancer (38). Other studies have revealed that $\mathrm{MTDH}$ plays an important regulatory role in tumor proliferation, differentiation, apoptosis, migration, invasion, and drug resistance (39-41) via the Ha-ras, PI3K/Akt, NFkappa B, and Wnt/beta-catenin signaling pathways $(38,41$, 42). MTDH is the target gene of miR-630. Zhao pointed out that miR-630 can directly target the MTDH gene. In the MDAMB-231-luc and BT549 breast cancer cell lines, high expression of miR-630 can inhibit cell movement and invasion (43).
However, whether other non-coding RNAs, such as circRNA, play a regulatory role in $\mathrm{MTDH}$ expression has not been ascertained. Our study screened out the miR-3662 that participates in MTDH regulation and can upregulate circRNA, and the upregulated circRNA was circ-03955. Subsequently, we validated the inhibitory effects of circ- 03955 on MTDH by reversing the inhibitory effect of miR-3662 through recovery experiments. miR-3662 can reverse the promotion of MTDH on osteosarcoma, and circ-03955 can reverse the inhibition of miR3662 on osteosarcoma, according to the recovery experiments. It was further demonstrated that circ-03955 and miR-3662 compete to regulate the expression of MTDH, thereby affecting the proliferation, apoptosis, and EMT of osteosarcoma.

In conclusion, our study shows that circ-03955 is highly expressed in osteosarcoma. Circ-03955 knockdown can inhibit the proliferation of osteosarcoma cells, promote cell apoptosis, and inhibit cell migration and invasion. Further analysis showed that circ-03955 was negatively correlated with miR-3662 in osteosarcoma, and circ-03955 could bind to miR-3662, indicating that there was a competitive relationship between circ-03955 and miR-3662 in the cell. Circ-03955 competes with miR-3662 to regulate the expression of MTDH and then affect the progression and occurrence of EMT in osteosarcoma. These findings provide a basis for potential therapeutic targets for the treatment of osteosarcoma.

\section{DATA AVAILABILITY STATEMENT}

The datasets presented in this study can be found in online repositories. The names of the repository/repositories and accession number(s) can be found in the article/supplementary material.

\section{ETHICS STATEMENT}

The animal study was approved by the medical ethics committee of the third Xiangya Hospital, central South University.

\section{AUTHOR CONTRIBUTIONS}

YZ designed the work and offered funds. ZW and MD designed the work, performed the experiment, and submitted the manuscript. LC and WW performed the experiment and collected the data. GL, DL, and $\mathrm{ZH}$ analyzed data and modified the manuscript. All authors contributed to the article and approved the submitted version.

\section{FUNDING}

This study was supported by the New Xiangya Talent Project of the Third Xiangya Hospital of Central South University (Grant NO. JY201516). 


\section{REFERENCES}

1. Maya K, Teng MW, Smyth MJ, Thomas DM. Translational biology of osteosarcoma. Nat Rev Cancer (2014) 14:722-35. doi: 10.1038/nrc3838

2. Simpson S, Dunning MD, De Brot S, Grau-Roma L, Mongan NP, Rutland CS. Comparative review of human and canine osteosarcoma: morphology, epidemiology, prognosis, treatment and genetics. Acta Vet Scand (2017) 59:71. doi: 10.1186/s13028-017-0341-9

3. Kovac M, Blattmann C, Ribi S, Smida J, Mueller NS, Engert F, et al. Exome sequencing of osteosarcoma reveals mutation signatures reminiscent of BRCA deficiency. Nat Commun (2015) 6:8940. doi: 10.1038/ncomms9940

4. Cai W, Wu B, Li Z, He P, Wang B, Cai A, et al. LncRNA NBR2 inhibits epithelial-mesenchymal transition by regulating Notch 1 signaling in osteosarcoma cells. J Cell Biochem (2018) 120:2015-27. doi: 10.1002/ jcb. 27508

5. Salzman J, Gawad C, Wang PL, Lacayo N, Brown PO. Circular RNAs are the predominant transcript isoform from hundreds of human genes in diverse cell types. PloS One (2012) 7:e30733. doi: 10.1371/journal.pone. 0030733

6. Guarnerio J, Zhang Y, Cheloni G, Panella R, Mae Katon J, Simpson M, et al. Intragenic antagonistic roles of protein and circRNA in tumorigenesis. Cell Res (2019) 29:628-40. doi: 10.1038/s41422-019-0192-1

7. Yao Y, Chen X, Yang H, Chen W, Qian Y, Yan Z, et al. Hsa_circ_0058124 promotes papillary thyroid cancer tumorigenesis and invasiveness through the NOTCH3/GATAD2A axis. J Exp Clin Cancer Res (2019) 38:318. doi: 10.1186/ s13046-019-1321-x

8. Jin J, Chen A, Qiu W, Chen Y, Li Q, Zhou X, et al. Dysregulated circRNA_100876 suppresses proliferation of osteosarcoma cancer cells by targeting microRNA-136. J Cell Biochem (2019) 120:15678-87. doi: 10.1002/ jcb. 28837

9. Wang L, Long H, Zheng Q, Bo X, Xiao X, Li B. Circular RNA circRHOT1 promotes hepatocellular carcinoma progression by initiation of NR2F6 expression. Mol Cancer (2019b) 18:119. doi: 10.1186/s12943-019-1046-7

10. Bonizzato A, Gaffo E, Te Kronnie G, Bortoluzzi S. CircRNAs in hematopoiesis and hematological malignancies. Blood Cancer J (2016) 6:e483. doi: 10.1038/ bcj.2016.81

11. Liang M, Huang G, Liu Z, Wang Q, Yu Z, Liu Z, et al. Elevated levels of hsa_circ_006100 in gastric cancer promote cell growth and metastasis via miR-195/GPRC5A signalling. Cell Prolif (2019) 52:e12661. doi: 10.1111/ cpr.12661

12. Chen G, Shi Y, Zhang Y, Sun J. CircRNA_100782 regulates pancreatic carcinoma proliferation through the IL6-STAT3 pathway. Onco Targets Ther (2017) 10:5783-94. doi: 10.2147/OTT.S150678

13. Li X, Sun XH, Xu HY, Pan HS, Liu Y, He L. Circ_ORC2 enhances the regulatory effect of miR-19a on its target gene PTEN to affect osteosarcoma cell growth. Biochem Biophys Res Commun (2019b) 514:1172-8. doi: 10.1016/ j.bbrc.2019.04.188

14. Xi Y, Fowdur M, Liu Y, Wu H, He M. Differential expression and bioinformatics analysis of circRNA in osteosarcoma. 39. Biosci Rep (2019) 39:5-14. doi: 10.1042/BSR20181514

15. Yanbin Z, Jing Z. CircSAMD4A accelerates cell proliferation of osteosarcoma by sponging miR-1244 and regulating MDM2 mRNA expression. Biochem Biophys Res Commun (2019) 516:102-11. doi: 10.1016/j.bbrc.2019.05.182

16. Ardila HJ, Sanabria-Salas MC, Meneses X, Rios R, Huertas-Salgado A, Serrano ML. Circulating miR-141-3p, miR-143-3p and miR-200c-3p are differentially expressed in colorectal cancer and advanced adenomas. Mol Clin Oncol (2019) 11:201-7. doi: 10.3892/mco.2019.1876

17. Ramirez-Moya J, Santisteban P. miRNA-Directed Regulation of the Main Signaling Pathways in Thyroid Cancer. Front Endocrinol (Lausanne) (2019) 10:430. doi: 10.3389/fendo.2019.00430

18. Li S, Pei Y, Wang W, Liu F, Zheng K, Zhang X. Circular RNA 0001785 regulates the pathogenesis of osteosarcoma as a ceRNA by sponging miR-1200 to upregulate HOXB2. Cell Cycle (2019a) 18:1281-91. doi: 10.1080/ 15384101.2019.1618127

19. Song YZ, Li JF. Circular RNA hsa_circ_0001564 regulates osteosarcoma proliferation and apoptosis by acting miRNA sponge. Biochem Biophys Res Commun (2018) 495:2369-75. doi: 10.1016/j.bbrc.2017.12.050
20. Sims PW, Vasser M, Wong WL, Williams PM, Meng YG. Immunopolymerase chain reaction using real-time polymerase chain reaction for detection. Analyt Biochem (2000) 281:230-2. doi: 10.1006/abio.2000.4578

21. Tang J, Shen L, Yang Q, Zhang C. Overexpression of metadherin mediates metastasis of osteosarcoma by regulating epithelial-mesenchymal transition. Cell Prolif (2014) 47:427-34. doi: 10.1111/cpr.12129

22. Ottaviani G, Jaffe N. The epidemiology of osteosarcoma. Cancer Treat Res (2009) 152:3. doi: 10.1007/978-1-4419-0284-9_1

23. Tan P, Zou C, Yong B, Ju H, Zhang L, Qiao S, et al. Expression and prognostic relevance of PRAME in primary osteosarcoma. Biochem Biophys Res Commun (2012) 419:801-8. doi: 10.1016/j.bbrc.2012.02.110

24. Mialou V, Philip T, Kalifa C, Perol D, Gentet JC, Marec-Berard P, et al. Metastatic osteosarcoma at diagnosis: prognostic factors and long-term outcome-the French pediatric experience. Cancer (2005) 104:1100-9. doi: 10.1002/cncr. 21263

25. Hughes DP. Strategies for the targeted delivery of therapeutics for osteosarcoma. Expert Opin Drug Delivery (2009) 6:1311-21. doi: 10.1517/ 17425240903280422

26. Zhang HD, Jiang LH, Sun DW, Hou JC, Ji ZL. CircRNA: a novel type of biomarker for cancer. Breast Cancer (2018) 25:1-7. doi: 10.1007/s12282-0170793-9

27. Wang L, Liang Y, Mao Q, Xia W, Chen B, Shen H, et al. Circular RNA circCRIM1 inhibits invasion and metastasis in lung adenocarcinoma through the microRNA (miR)-182/miR-93-leukemia inhibitory factor receptor pathway. Cancer Sci (2019a) 110:2960-72. doi: 10.1111/ cas. 14131

28. Yu J, Yang M, Zhou B, Luo J, Zhang Z, Zhang W, et al. CircRNA-104718 acts as competing endogenous RNA and promotes hepatocellular carcinoma progression through microRNA-218-5p/TXNDC5 signaling pathway. Clin Sci (2019) 133:1487-503. doi: 10.1042/CS20190394

29. Liu YC, Li JR, Sun CH, Andrews E, Chao RF, Lin FM, et al. CircNet: a database of circular RNAs derived from transcriptome sequencing data. Nucleic Acids Res (2016) 44:D209-215. doi: 10.1093/nar/gkv940

30. Li Z, Huang C, Bao C, Chen L, Lin M, Wang X, et al. Exon-intron circular RNAs regulate transcription in the nucleus. Nat Struct Mol Biol (2015) 22:256-64. doi: 10.1038/nsmb.2959

31. Chen Z, Zuo X, Zhang Y, Han G, Zhang L, Wu J, et al. MiR-3662 suppresses hepatocellular carcinoma growth through inhibition of HIF-1alphamediated Warburg effect. Cell Death Dis (2018) 9:549. doi: 10.1038/ s41419-018-0616-8

32. Hansen TB, Jensen TI, Clausen BH, Bramsen JB, Finsen B, Damgaard CK, et al. Natural RNA circles function as efficient microRNA sponges. Nature (2013a) 495:384-8. doi: 10.1038/nature11993

33. Memczak S, Jens M, Elefsinioti A, Torti F, Krueger J, Rybak A, et al. Circular RNAs are a large class of animal RNAs with regulatory potency. Nature (2013) 495:333-8. doi: 10.1038/nature11928

34. Hansen TB, Kjems J, Damgaard CK. Circular RNA and miR-7 in cancer. Cancer Res (2013b) 73:5609-12. doi: 10.1158/0008-5472.CAN-13-1568

35. Capel B, Swain A, Nicolis S, Hacker A, Walter M, Koopman P, et al. Circular transcripts of the testis-determining gene Sry in adult mouse testis. Cell (1993) 73:1019-30. doi: 10.1016/0092-8674(93)90279-Y

36. Fang Y, Xue JL, Shen Q, Chen J, Tian L. MicroRNA-7 inhibits tumor growth and metastasis by targeting the phosphoinositide 3-kinase/Akt pathway in hepatocellular carcinoma. Hepatology (2012) 55:1852-62. doi: 10.1002/ hep. 25576

37. Zhao X, Dou W, He L, Liang S, Tie J, Liu C, et al. MicroRNA-7 functions as an anti-metastatic microRNA in gastric cancer by targeting insulin-like growth factor-1 receptor. Oncogene (2013) 32:1363-72. doi: 10.1038/onc.2012.156

38. Hu G, Wei Y, Kang Y. The multifaceted role of MTDH/AEG-1 in cancer progression. Clin Cancer Res (2009) 15:5615-20. doi: 10.1158/10780432.CCR-09-0049

39. Li X, Kong X, Huo Q, Guo H, Yan S, Yuan C, et al. Metadherin enhances the invasiveness of breast cancer cells by inducing epithelial to mesenchymal transition. Cancer Sci (2011) 102:1151-7. doi: 10.1111/j.1349-7006.2011. 01919.x

40. Liu X, Zhang N, Li X, Moran MS, Yuan C, Yan S, et al. Identification of novel variants of metadherin in breast cancer. PloS One (2011) 6:e17582. doi: 10.1371/journal.pone.0017582 
41. Song E, Yu W, Xiong X, Kuang X, Ai Y, Xiong X. Astrocyte elevated gene-1 promotes progression of cervical squamous cell carcinoma by inducing epithelial-mesenchymal transition via Wnt signaling. Int J Gynecol Cancer (2015) 25:345-55. doi: 10.1097/IGC.0000000000000381

42. Zhu GC, Yu CY, She L, Tan HL, Li G, Ren SL, et al. Metadherin regulation of vascular endothelial growth factor expression is dependent upon the PI3K/ Akt pathway in squamous cell carcinoma of the head and neck. Med (Baltimore) (2015) 94:e502. doi: 10.1097/MD.0000000000000502

43. Zhou CX, Wang CL, Yu AL, Wang QY, Zhan MN, Tang J, et al. MiR-630 suppresses breast cancer progression by targeting metadherin. Oncotarget (2016) 7:1288-99. doi: 10.18632/oncotarget.6339
Conflict of Interest: The authors declare that the research was conducted in the absence of any commercial or financial relationships that could be construed as a potential conflict of interest.

Copyright (C) 2020 Wang, Deng, Chen, Wang, Liu, Liu, Han and Zhou. This is an open-access article distributed under the terms of the Creative Commons Attribution License (CC BY). The use, distribution or reproduction in other forums is permitted, provided the original author(s) and the copyright owner(s) are credited and that the original publication in this journal is cited, in accordance with accepted academic practice. No use, distribution or reproduction is permitted which does not comply with these terms. 